\title{
Web Fusion Model of Internet of Things Based on Hierarchy Requirement Analysis
}

\author{
YANG Hui-jun ${ }^{1}$ \\ 1. Department of Information Service, Anhui Institute of International Business, \\ Hefei AnHui, 231131, China \\ yhj997381@163.com
}

\begin{abstract}
Each item of sensory information is effectively integrated by Internet of things (IoT) system. However, due to different framework and interface, software and hardware platform and communication protocol are mutually independent, which makes distributed IoT system unable to fulfill uniform operation and management. By aiming at issues difficultly fulfilled by IoT system, a kind of framework model of IoT system based on Web Service is put forward. Firstly, function and framework analysis is conducted for local sensor service system, integrated sensor service system and integrated application service system required by model; next, an introduction about design of Web Service interface is made; finally, services and functions are abstracted to normalize the relation among three varieties of service systems of model. This scheme is proved to be with good applicability, flexibility and expansibility in cases.
\end{abstract}

Keywords: Web service; IoT system; Distributed type; Framework mode; Integration

\section{Introduction}

As one of five major emerging strategic industries in China, IoT has been increasingly attached to importance by all sectors of society. With rapid development of global information superhighway, Internet receives and handles massive information on a daily basis. Being able to include properties of objects in information sharing of Internet will greatly enhance social productive forces and will also promote overall development of society. Basic definition of IoT is namely a network where sensing device is embedded in various objects and generally associated as objects. This definition clearly shows two important links in development of IoT, namely sensor technology and network association [1]. Sensor technology has already used in computer field; for example, radio frequency identification technology has been widely applied to fields such as logistics management and shopping in supermarket. A mature IoT system is required to effectively integrate each item of sensory information and handle all kinds of information at any time. Besides, the system is able to be highly flexible to enhance its expansibility and also able to prevent damage of some subsystems and sub-nodes from having disastrous impacts on overall system [2]. A variety of IoT system framework based on Web Service technology is researched in this article. Through application of such technology, functions and methods are efficiently integrated [4] to fulfill date sharing and collaboration in heterogeneous platform and multi-platform [2,3]. Each subsystem is uniformly managed and integrated by application of service release, request and supply $[5,6]$.

\section{Overall Structure Design}

IoT system framework model based on Web Service mainly includes three parts: Local Sensor Subsystem (referred to as LS system), Integrated Sensor Service Subsystem 
(referred to as ISS system) and Integrated Application Subsystem (referred to as IA system), as showed in Figure. 1.

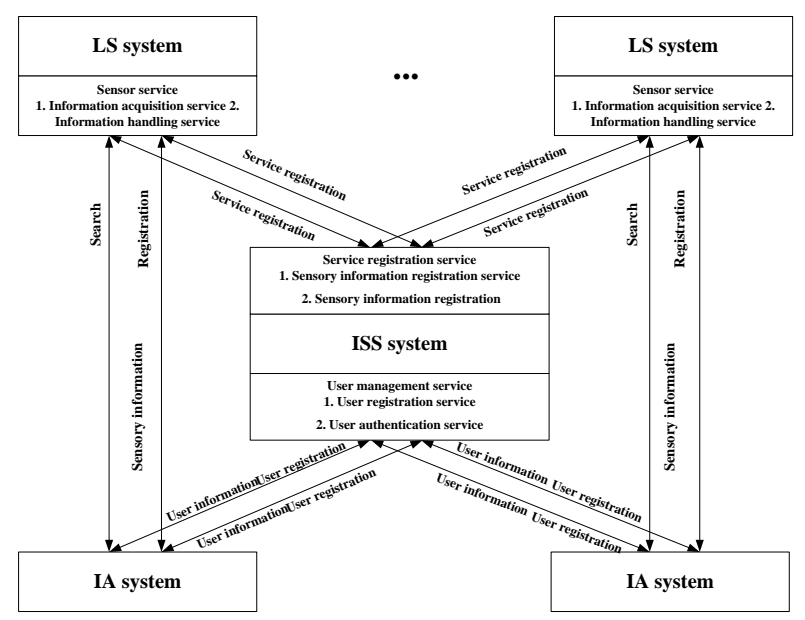

Figure 1. Overall Topological Structure of System

\subsection{LS System}

As a provider of application service, LS system provides IA system and ISS system data information. Generally, corresponding to one LS system, each sensing detection area uses various smart sensor devices to gather required device data or environmental information, store such data or transmit it to target storer by wireless communication and other modes [7]. LS system can provide service release function, accept corresponding service request and handle corresponding date request at the same time.

\subsection{ISS System}

ISS system fulfills centralized management for sensor service and is able to accept service registration of LS system, provide IA system with corresponding access index and address and conducts uniform maintenance and management for users of overall system. Besides, as an administrator of services, ISS system is able to associate LS system with IA system.

\subsection{IA system}

As a user of sensor service, IA system makes service request by integration of LSS system [10]. Prior to each usage of service, user authentication of ISS system is required to be passed; available services provided by LS system can be searched by authorized request; a request is made to it and corresponding service response is obtained.

\section{Module Design of Subsystem}

\subsection{Structure of LS System}

Main module functions of LS system are as shown in Figure. 2, mainly including three main functional modules, network communication, data handling and sensing terminal. 
LS system

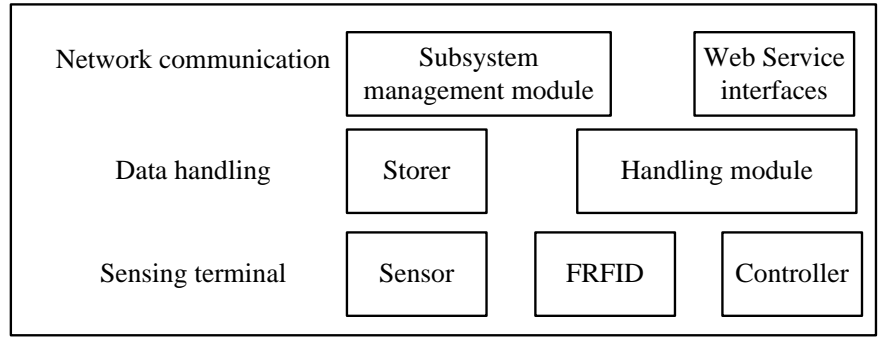

Figure 2. Structure of LS System

3.1.1. Sensing Terminal: Sensing terminal is located at lowest end of system and used to sense corresponding sensing data. Generally, sensing terminal includes sensor, RFID and controller used to manage sensor device [8,9]. Controller provides sensing nodes with energy source and data control in most cases. Requirements for sensing terminal shall fully accommodate to data requirement of system so that effective interaction can be conducted with higher data handling level.

3.1.2. Data Handing: Main function of data handling is to store date and basically handle original date so as to acquire original date provided by sensing terminal and provide subsystem management module with primary product data [12]. Date handing impacts form of data acquired by LS system

3.1.3. Network Communication: Main function of network communication is to acquire data of sensing terminal and send such data to other systems for application in form of service after handling it. One uniform management module is included in most cases and used to manage lower data storage device and etc [13]. Data and service interaction among subsystems are conducted in this system by means of web service interface.

\subsection{ISS system}

For the purpose that sensor services are able to be uniformly integrated and used conveniently and safely, ISS system is designed. As an administrator of sensor services, ISS system mainly includes three functional modules, service management, user management and module management, as shown in Figure 3.

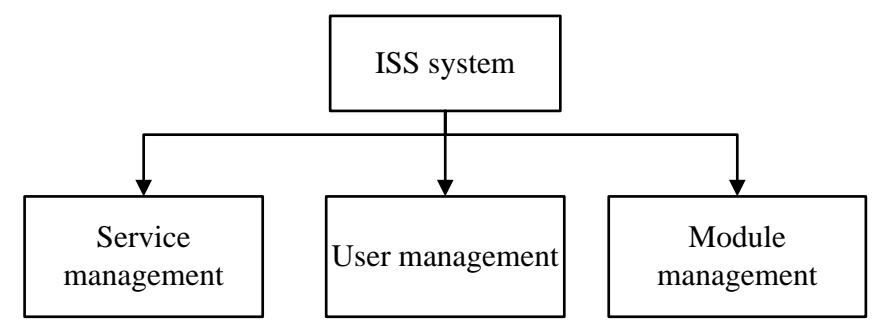

Figure 3. Structure of ISS System

3.2.1. Service Management: Service management is used to uniformly manage functional services of system. Each LS system is able to register services by means of data service interfaces provided by service management module. By these global indexes, required service interfaces can be searched in IA system. After request works, data access, resource management and device control can be fulfilled for LS system. Service management module enhances flexibility of system. Services can be effectively added and 
deleted only with indexes. Thus, the system is made to be equipped with better expansibility.

3.2.2. User Management: User management module is mainly used to manage users, roles, authority and log. By management function of this module, information about users is intensively controlled to make it convenient for multi-data and service source system to be effectively integrated. Roles are generally associated with various software functional modules. Each registered user will correspond to one corresponding role. The system identifies different authorities mainly by means of different roles. Authority management is to divide overall system on the basis of modules and grant one or one group of authorities to each module. Such module fulfills uniform access authorization at the same time. The system records users' operation by log management so as to trace operation records for overall system.

3.2.3. Module Management: Aiming at various functional modules of system, module management function divides modules on the basis of type. There is different management strategy for each type of module. Interface module is special for same functional module is used for one group of similar web service interfaces. If web service interface of different type is added to the system, corresponding functional module is required to be applied for so as to handle data and provide the system with corresponding data information.

\subsection{IA System}

IA system is used by users in the form of uniform client software or web browsing in most cases. IA system is a user of services. By requesting corresponding services from ISS system, authorized users can acquire corresponding date of corresponding services after calling in accordance with functional parameters set in LS system. IA system includes four parts, service application, data conversion, and application module and system management, as showed in Figure 4.

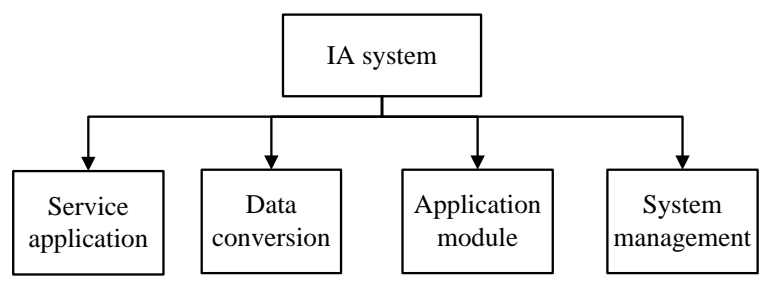

Figure 4. Structure of IA System

3.3.1. Service Application: Service application is used to send request information to ISS system and acquire corresponding feedback information. Service application reads global service index and provides users with name of corresponding services and information about parameters. Users can call corresponding Web Service after obtaining such information.

3.3.2. Data Conversion: To make it convenient for information read from Web Service to be correctly used for local service requester, data conversion functional module is added in front of specific application module in most cases. Data information provided by web service can be freely combined and purposefully selected by such module [5] so that the system is able to use more flexible data information, which provides the system with more function outputs. 
3.3.3. Application Module: Application module provides specific application demonstration, which is designed on the basis of different users' demand in most cases. Application module acquires data format suitable for module by data conversion and brings convenience to users' usage by visual page demonstration. For C/S system, fulfillment is conducted by calling different elements in most cases; for B/S system, by calling different page URL.

3.3.4. System Management: System management provides management functions for IA system, such as module management and data format management. System management functions can be designed on the basis of different operation demand. If services provided by LS system are required to be used, service application shall also be carried out.

\section{Technical Application of Web Service}

Web Service is loosely coupled, self-contained and programmable web application program mutually independent from platform. It provides users with function and method by network; and such provision is remote and heterogeneous. Web Service has been widely applied to development of distributed system at present for it provides a kind of flexible and powerful integration mechanism and combines superiority similar to elements. Web service provides an advanced distribution computation model and provides heterogeneous system with highly efficient mechanism of data and information integration. Supporting technology of Web Service system is used to make Web Service equipped with characteristics including application-oriented, highly integrated and highly comprehensive and make developers focus more on design of overall system framework. For combination between Web Service and existing platform, characteristics of existing system framework and various function of existing system shall be fully analyzed to reasonably develop levels of module.

IoT application system has the characteristics of distributed type in most cases. System modules shall be reasonably divided on the basis of full integration with characteristics of web service; and internal structure of each module shall be reasonably planned. In accordance with characteristics of IoT system, three roles, data provider, data user and data administrator, shall be classified in module. Meanwhile, service function and data handing are combined to put forward reasonable system framework by combination with several processes of web service.

\section{Service Interface Design}

As a service-oriented one, Web Service interface directly performs service integration for functions. Service process is fulfilled by XMl, including service description, service registration, service release, and service request and service feedback. Transport protocol of Web Service is HTTP, which makes it easier to access resource and return to results. Web Service standards are developed by W3C, of which the greatest feature is irrelevance with platform. Namely, work process of Web Service is irrelevant with operation system, operation platform and the like by open protocol. Such irrelevance with platform makes this technology facilitate service interaction and service integration in heterogeneous platform more. In addition, Web Service fulfills dynamic integration by UDDI, which is able to fulfill discovery, binding and application of dynamic services. By comparison with static integration scheme (such as API), this dynamic scheme enhances flexibility of system, facilitate expansion of system and make adaptability of overall system greatly enhanced.

In consideration of universality and practicability of this system framework model, we put forward interface models for three subsystems as shown in Table 1. 
Table 1. Web Service Interface Design

\begin{tabular}{cccc}
\hline Name & Provider & Service object & Function \\
\hline $\begin{array}{c}\text { Information acquisition } \\
\text { service }\end{array}$ & LS system & ISS system & Providing sensory information \\
$\begin{array}{c}\text { Information handling } \\
\text { service }\end{array}$ & LS system & ISS system & Handing sensory information \\
$\begin{array}{c}\text { Service interface } \\
\text { registration }\end{array}$ & ISS system & LS system & $\begin{array}{c}\text { Providing service interface } \\
\text { registration }\end{array}$ \\
$\begin{array}{c}\text { User registration } \\
\text { service }\end{array}$ & ISS system & IA system & Providing user registration \\
$\begin{array}{c}\text { User authentication } \\
\text { service }\end{array}$ & ISS system & IA system & Providing user authentication \\
\hline
\end{tabular}

\subsection{LS System Interface Design}

LS system interfaces include information acquisition interface and information handling interface, which are used to complete acquisition and handling of information. Information acquisition interface provides information about sensor device, information about sensory information source and information about sensor data. Information handling service provides management and handing of information and controls turning on and off and discarding of sensor device.

\subsection{ISS system Interface Design}

ISS system interfaces can be divided into two types: one is oriented to registration service provided by LS system; another is oriented to user management service interface provided by IA system. Service registration interfaces are namely registration and cancellation of service interfaces. Registered service can be retrieved by service index and remind users of name, function, parameters and return value and address of services. Service authentication includes user registration service and user authentication service. User registration service includes service index services, service authorization and user registration service. Service registrants are provided with all available service lists by service index services. Service registrants' applying service is fulfilled by service authorization. Information about users is added to user list by user registration service to make it convenient for user management. Information about registration services generally covers ID of user, password, user name, user authority and etc. Management is fulfilled for user authority and system login log by user authentication service. Authentication information covers ID of user, password, login time and etc. The system will display different service indexes for registered and authenticated users in accordance their different authorities.

\section{Model Application}

To verify feasibility of this model, effectiveness of the scheme above is verified by software development for some plant room system and operation conditions in practical environment. LS system is set in all plant rooms to monitor temperature and humidity environment, emergency power supply, power supply, water leakage, regulated power supply and other items, as shown in Figure 5. 


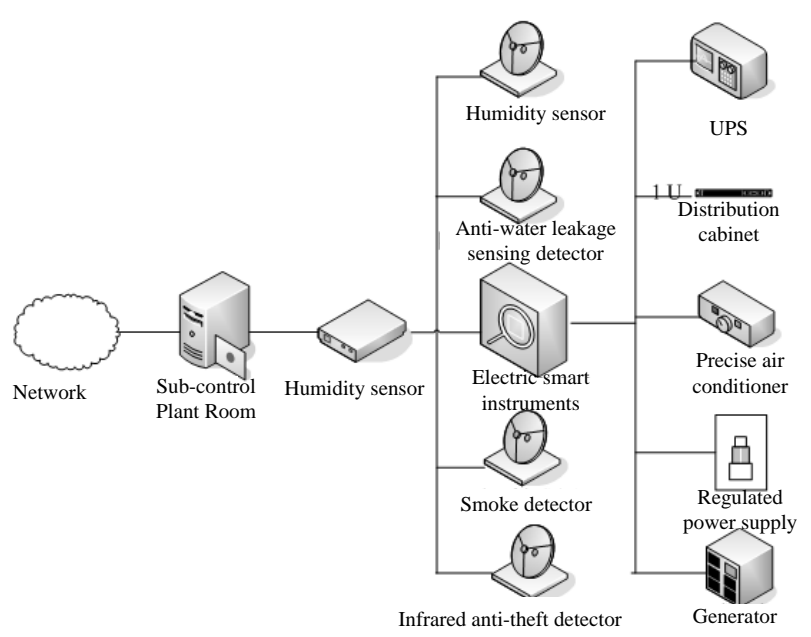

Figure 5. Monitoring Structure of Plant Room

ISS system is placed at Central Plant Room and connected with LS system of Subcontrol Plant Room by Internet. ISS system is able to fulfill effective management and control for all Sub-control Plant Rooms and real-time acquire availability of system of each Sub-control Plant Room. ISS system provides plant room management personnel with user list function, which able to assign different authorities for personnel in accordance with their roles. For example, power supply management personnel for plant room are able to monitor conditions of electric instruments only.

ISS system can be placed at any place. Multi IA systems can be set. Only registration is required in ISS system for each addition. ISS system can be released by client end or in the form of webpage of browser and also can have different display interfaces for different plant room management personnel. For example, plant room manager is required to observe daily statement of various detection data only; electrical and mechanical maintenance personnel are required to observe power supply conditions in due time. Topological structure of overall system is as shown in Figure 6.

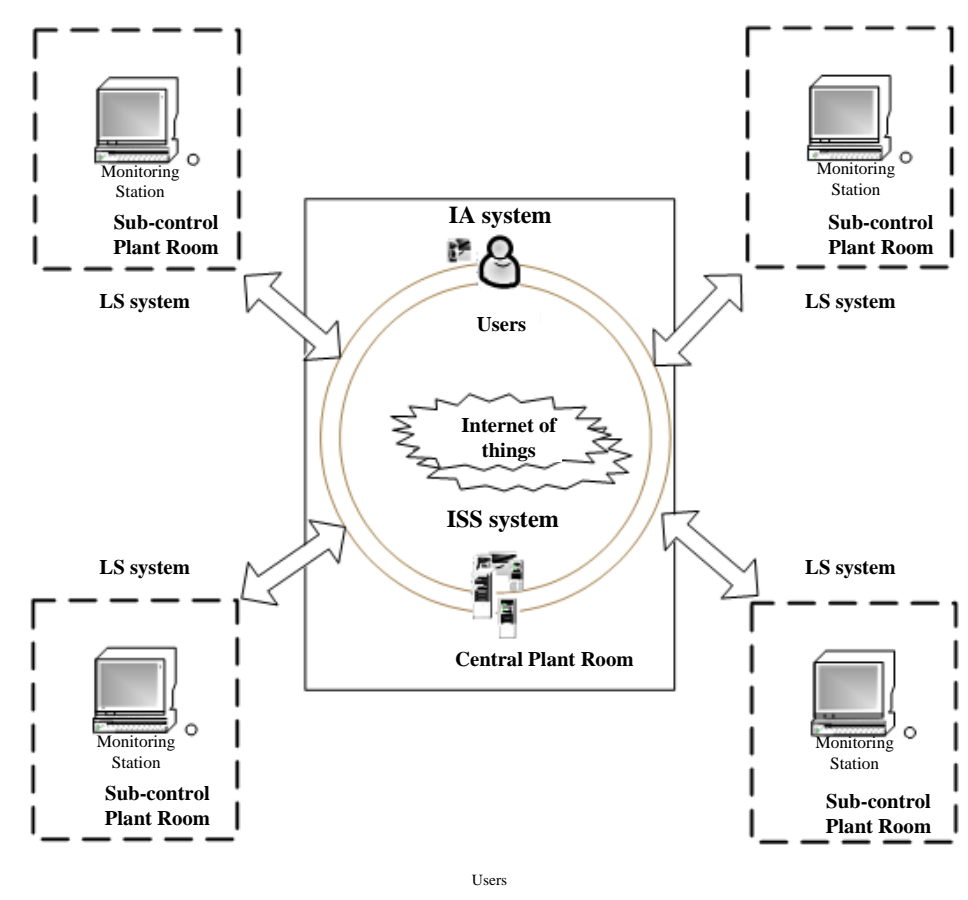

Figure 6. Topological Structure of System 


\section{Conclusions}

A kind of system framework model based on Web Service is put forward for distributed IoT system in this article. Three varieties of service systems required by model are put forward: LS system, ISS system and IA system. And analysis is conducted for function and framework of each system. Web Service interface is the most important aspect of system. Further analysis is conducted for interfaces provided by each subsystem in this article. The model is verified and applied by a practical design case in end of this article. Services and functions are abstracted to normalize the relation among three varieties of service systems of model in this scheme, which is with good applicability. Besides, cross-platform characteristics of Web Service make this model able to be more consistent with practical conditions of distributed system and enhance flexibility and expansibility of system. This system performs centralized management for services and users by ISS system and plays an important role in normalization and efficient execution of services. There are still many deficiencies in this scheme. For example, how to further increase reusability of system, extract system elements more reasonably and design reasonable database and data sheet structure. these issues will be theme of next research.

\section{Acknowledgement}

The research projects of major educational reform of higher education in Anhui Province under Grant No. 2014-zdjy177; the funded projects of education revitalization plan for higher vocational professional leaders ,2014, in Anhui Province.

\section{Reference}

[1] Degui Zeng, Yishuang Geng, Content distribution mechanism in mobile P2P network, Journal of Networks, 9(5), 1229-1236, Jan. (2014).

[2] Gu W, Lv Z, Hao M. Change detection method for remote sensing images based on an improved Markov random field[J]. Multimedia Tools and Applications, (2015): 1-16.

[3] Chen Z, Huang W, Lv Z. Towards a face recognition method based on uncorrelated discriminant sparse preserving projection[J]. Multimedia Tools and Applications, (2015): 1-15.

[4] Jinyu Hu and Zhiwei Gao. Distinction immune genes of hepatitis-induced heptatocellular carcinoma[J]. Bioinformatics, (2012), 28(24): 3191-3194.

[5] Su T, Wang W, Lv Z, et al. Rapid Delaunay triangulation for randomly distributed point cloud data using adaptive Hilbert curve[J]. Computers \& Graphics, (2016) 54: 65-74.

[6] Gu W, Lv Z, Hao M. Change detection method for remote sensing images based on an improved Markov random field[J]. Multimedia Tools and Applications, (2015): 1-16.

[7] Lv Z, Tek A, Da Silva F, et al. Game on, science-how video game technology may help biologists tackle visualization challenges[J]. PloS one, (2013), 8(3): 57990.

[8] Chen Z, Huang W, Lv Z. Towards a face recognition method based on uncorrelated discriminant sparse preserving projection[J]. Multimedia Tools and Applications, (2015): 1-15.

[9] Jiang D, Ying X, Han Y, et al. Collaborative multi-hop routing in cognitive wireless networks[J]. Wireless Personal Communications, (2015): 1-23.

[10] Lv Z, Tek A, Da Silva F, et al. Game on, science-how video game technology may help biologists tackle visualization challenges[J]. PloS one, (2013), 8(3): 57990.

[11] Jiang D, Xu Z, Lv Z. A multicast delivery approach with minimum energy consumption for wireless multi-hop networks[J]. Telecommunication Systems, (2015): 1-12.

[12] Fu C, Zhang P, Jiang J, et al. A Bayesian approach for sleep and wake classification based on dynamic time warping method[J]. Multimedia Tools and Applications, (2015): 1-20.

[13] Lv Z. Wearable smartphone: Wearable hybrid framework for hand and foot gesture interaction on smartphone[C]//Computer Vision Workshops (ICCVW), 2013 IEEE International Conference on. IEEE, (2013): 436-443.

[14] Lin Y, Yang J, Lv Z, et al. A Self-Assessment Stereo Capture Model Applicable to the Internet of Things[J]. Sensors, (2015), 15(8): 20925-20944. 


\section{Author}

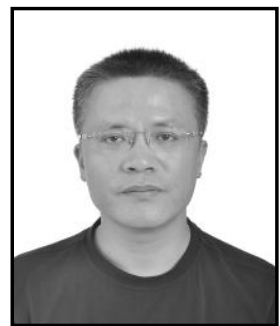

Yang Huijun. Yang Huijun, Male, was born on August 1, 1973, in Yuexi Anhui, China. associate professor and Master of the department of Information Service of the Anhui Institute of International Business . The research field : the Internet of things application technology, intelligent learning environment. 
International Journal of Smart Home

Vol. 10, No. 6 (2016) 